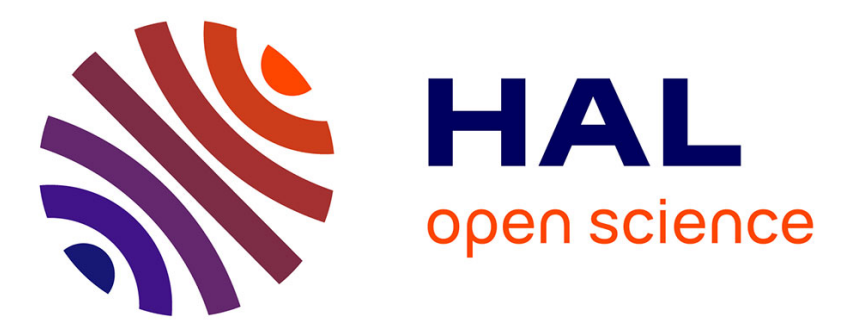

\title{
Strongly Structural Input and State Observability for Linear Time Invariant Network Systems
}

\author{
Sebin Gracy, Federica Garin, Alain Kibangou
}

\section{To cite this version:}

Sebin Gracy, Federica Garin, Alain Kibangou. Strongly Structural Input and State Observability for Linear Time Invariant Network Systems. ECC 2019 - 18th European Control Conference, Jun 2019, Naples, Italy. pp.2516-2521, 10.23919/ECC.2019.8796307 . hal-02094223

\section{HAL Id: hal-02094223 https://hal.inria.fr/hal-02094223}

Submitted on 9 Apr 2019

HAL is a multi-disciplinary open access archive for the deposit and dissemination of scientific research documents, whether they are published or not. The documents may come from teaching and research institutions in France or abroad, or from public or private research centers.
L'archive ouverte pluridisciplinaire HAL, est destinée au dépôt et à la diffusion de documents scientifiques de niveau recherche, publiés ou non, émanant des établissements d'enseignement et de recherche français ou étrangers, des laboratoires publics ou privés. 


\title{
Strongly Structural Input and State Observability for Linear Time Invariant Network Systems
}

\author{
Sebin Gracy, Federica Garin, and Alain Y. Kibangou
}

\begin{abstract}
This paper considers linear discrete-time network systems affected by unknown inputs, studying whether they are input and state observable (ISO), namely whether both the initial state and the unknown input can be reconstructed from the output measurements. More precisely, the paper studies ISO, where the input reconstruction must happen with at most one time-step delay, and unconstrained ISO, where a larger delay is allowed. The focus is on strongly structured (unconstrained) ISO, wherein the objective is to find conditions such that for all system matrices that share a given zero/nonzero pattern, the resulting system is (unconstrained) ISO. We first provide a graphical characterization for strongly structural unconstrained ISO. Then, we find a sufficient condition and a necessary condition for strongly structural ISO, which are equivalent in the case without direct feedthrough. All conditions are in terms of existence of suitable uniquely restricted matchings in bipartite graphs associated with the system.
\end{abstract}

\section{INTRODUCTION}

Graphical characterizations for system-theoretic properties of network systems have the advantage that they rely only on the network structure, while knowledge of the actual entries in the system matrices is not essential, unlike algebraic characterizations. The notion of structured systems, i.e. systems whose matrices of the state-space realization have positions that are fixed to zero, forms the underpinning for graphical characterizations. In structured systems, the positions which are not a priori fixed to zero are free parameters. Under such a setting, one line of investigation focuses on strongly structural (s-structural) results, which insists that a property holds for all non-zero parameters, while another line of research studies generic results, which only ensure that a property holds true for almost all parameters. The present paper is focused on s-structural results.

Graph-theoretical characterizations for s-structural controllability (which, by duality, give corresponding results for observability) have been studied since [1]. Recent results on s-structural controllability have been provided in [2], [3]. Their graphical characterizations are in terms of uniquely restricted matchings in appropriately defined bipartite graphs. Another line of work, instead, characterizes s-structural controllability using the notion of zero forcing sets [4], [5]. The equivalence of the two approaches has been shown in [5].

The dynamics of a network system could be affected by unknown inputs. Such inputs might represent a malicious external attacker in a cyber-physical security problem, or a fault, or the effect of a subsystem with unmodeled dynamics.

The authors are with Univ. Grenoble Alpes, INRIA, CNRS, GrenobleINP, GIPSA-lab, F-38000 Grenoble, France. E-mail: sebin.gracy@inria.fr, federica.garin@inria.fr, alain.kibangou@univ-grenoble-alpes.fr
In order to ensure system monitoring and possibly to counteract the effect of the input to maintain proper functioning, it is desirable to be able to reconstruct both the state and the unknown input from the outputs. The possibility to do such reconstruction is referred to as Input and State $\mathrm{Ob}$ servability (ISO). More precisely, with ISO we will indicate the capability to reconstruct the initial state and the unknown inputs, insisting that the inputs are reconstructed with a delay of one time-step only. With unconstrained ISO, instead, we will allow the reconstruction of the unknown inputs to have arbitrary delay, possibly larger than one.

The problem of finding graphical characterizations for sstructural ISO has been addressed in [6], [7] under different settings. The results in [6] concern both LTI network systems and linear time-varying (LTV) network systems with fixed graphs, but are reliant on the following restrictive assumptions: a) each unknown input acts on exactly one state; b) each state is acted upon by at most one unknown input; c) direct measurements of a few states are available; and d) no direct feedthrough. On the other hand, the setting in [7] concerns LTV systems only, but is more general than that in [6] in the sense that time-varying graphs are accounted for and no assumptions are made on the structure of system matrices. In the particular case of an LTV system with timeinvariant graph, the sufficient condition in [7, Theorem 21)] for s-structural ISO of the LTV system is a sufficient condition also for s-structural ISO of the LTI system with the same graph. Indeed, this condition ensures that the system with such network structure is ISO for all possible nonzero parameters, irrespective of how they vary in time, and hence in particular for all time-invariant ones. However, this condition involves the so-called dynamic bipartite graph over a time interval of length $N$ (where $N$ is the dimension of the state space), whose number of vertices grows quadratically with $N$. Moreover, this is only a sufficient condition, and there is no simple way to derive a necessary condition for the LTI case from the LTV results in [7].

The main contribution of the present paper is to give graphical conditions for s-structural ISO of LTI systems, without the restrictive assumptions considered in [6], involving smaller graphs than the dynamic bipartite graph considered in [7], and including both a sufficient and a necessary condition. Our main results are a characterization of s-structural unconstrained ISO (Theorem 1), and then a sufficient condition and a necessary condition for ISO (Theorem 2), which are equivalent in the case of systems without direct feedthrough, where they give a characterization of sstructural ISO (Corollary 1). 


\section{Problem Formulation}

Consider a discrete-time LTI network system, whose dynamics are given by:

$$
\left\{\begin{aligned}
x_{k+1} & =A x_{k}+B u_{k} \\
y_{k} & =C x_{k}+D u_{k}
\end{aligned}\right.
$$

with state vector $x \in \mathbb{R}^{N}$, unknown input vector $u \in \mathbb{R}^{P}$ and output vector $y \in \mathbb{R}^{M}$. Matrices $A B, C$ and $D$ have positions that are a priori fixed to zero, and free parameters (that is, distinct real-valued parameters, which can be chosen arbitrarily) in all other positions. The position of zeros represents the interactions that cannot happen in this network system, while parameters represent the intensity of existing interactions.

In this paper, we will study input and state observability (ISO), according to the following two definitions.

Definition 1: System (1) is unconstrained ISO if there exists some integer $\ell \geq 0$ such that the initial condition $x_{0}$ and the unknown inputs $u_{0}, u_{1}, \ldots, u_{N-\ell}$ can be uniquely determined from the outputs $y_{0}, y_{1}, \ldots, y_{N}$.

While unconstrained ISO does not insist on a particular value of $\ell$, the following definition of ISO insists that the unknown input be reconstructed with delay 1 .

Definition 2: System (1) is ISO if the initial condition $x_{0}$ and the unknown inputs $u_{0}, u_{1}, \ldots, u_{N-1}$ can be uniquely determined from the measured outputs $y_{0}, y_{1}, \ldots, y_{N}$.

The goal of this paper is to study which positions of zeros in matrices $A, B, C, D$ ensure that the system is (unconstrained) ISO, irrespective of the value of the parameters, provided each parameter is non-zero. In other words, we will study strongly structural (s-structural) unconstrained ISO and ISO, defined as follows.

Definition 3: The structured system (1) is s-structurally ISO (resp. s-structurally unconstrained ISO) if the system is ISO (resp. unconstrained ISO) for all non-zero parameters. Our results will be based on the graphical descriptions of the zero positions that will be introduced in Sect. III-A.

\section{GRAPH CONSTRUCTIONS}

\section{A. Graphs Associated with the Structured System}

The structured system (1) is usually described by a directed graph $\mathcal{G}$, whose edges correspond to the entries of matrices $A, B, C$, and $D$ which are free parameters. The vertex set of $\mathcal{G}$ is $U \cup X \cup Y$, where $U=\left\{u_{1}, \ldots, u_{P}\right\}$, $X=\left\{x_{1}, \ldots, x_{N}\right\}$ and $Y=\left\{y_{1}, \ldots, y_{M}\right\}$ are the input, state and output vertex sets, respectively. The edge set of $\mathcal{G}$ is $\mathcal{E}_{A} \cup \mathcal{E}_{B} \cup \mathcal{E}_{C} \cup \mathcal{E}_{D}$, where:

- $\mathcal{E}_{A}=\left\{\left(x_{j}, x_{i}\right) \in X \times X\right.$ s.t. $\left.A_{i j} \neq 0\right\}$,

- $\mathcal{E}_{B}=\left\{\left(u_{j}, x_{i}\right) \in U \times X\right.$ s.t. $\left.B_{i j} \neq 0\right\}$,

- $\mathcal{E}_{C}=\left\{\left(x_{j}, y_{i}\right) \in X \times Y\right.$ s.t. $\left.C_{i j} \neq 0\right\}$, and

- $\mathcal{E}_{D}=\left\{\left(u_{j}, y_{i}\right) \in U \times Y\right.$ s.t. $\left.D_{i j} \neq 0\right\}$.

In this paper, instead of $\mathcal{G}$, we will rather use another description of the structured system, with the following bipartite graph. The bipartite graph $\mathcal{H}$ has left vertex set $U \cup X$ and right vertex set $X^{\prime} \cup Y$, where $U, X$, and $Y$ have the same definition as in the construction of $\mathcal{G}$, and

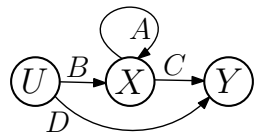

(a)

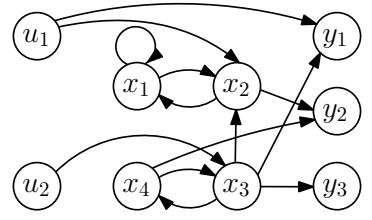

(b)
Fig. 1: Directed graph $\mathcal{G}$. (a) Sketch of its construction; (b) Example 1.

$X^{\prime}=\left\{x_{1}^{\prime}, \ldots, x_{N}^{\prime}\right\}$ is a copy of the state vertex set $X$. The edge set of $\mathcal{H}$ is $\overline{\mathcal{E}}_{A} \cup \overline{\mathcal{E}}_{B} \cup \overline{\mathcal{E}}_{C} \cup \overline{\mathcal{E}}_{D}$, where:

- $\overline{\mathcal{E}}_{A}=\left\{\left(x_{j}, x_{i}^{\prime}\right) \in X \times X^{\prime}\right.$ s.t. $\left.A_{i j} \neq 0\right\}$,

- $\overline{\mathcal{E}}_{B}=\left\{\left(u_{j}, x_{i}^{\prime}\right) \in U \times X^{\prime}\right.$ s.t. $\left.B_{i j} \neq 0\right\}$,

- $\overline{\mathcal{E}}_{C}=\left\{\left(x_{j}, y_{i}\right) \in X \times Y\right.$ s.t. $\left.C_{i j} \neq 0\right\}$, and

- $\overline{\mathcal{E}}_{D}=\left\{\left(y_{j}, u_{i}\right) \in U \times Y\right.$ s.t. $\left.D_{i j} \neq 0\right\}$.

Similarly to $\mathcal{G}$, also $\mathcal{H}$ fully describes the structured system, since its edges are in one-to-one correspondence with the non-zero entries of matrices $A, B, C$, and $D$.

In addition to $\mathcal{H}$, the statement of our main results in Sect. IV will also involve some other bipartite graphs $\mathcal{H}_{\times}$, $\mathcal{N}, \tilde{\mathcal{N}}$, and $\mathcal{N}_{0}$, whose definitions are given below, and whose meaning is unveiled in the proofs in Sections $\mathrm{V}$ and VI.

The bipartite graph $\mathcal{H}_{\times}$has the same vertex set as $\mathcal{H}$, and edge set which is a suitable extension of the edge set of $\mathcal{H}$, that will be defined below. We will use the word "self-loops" to indicate edges $\left(x_{i}, x_{i}^{\prime}\right) \in X \times X^{\prime}$, since they correspond to actual self-loops $\left(x_{i}, x_{i}\right)$ in $\mathcal{G}$. We define $\mathcal{E}_{\text {loop }} \subseteq \overline{\mathcal{E}}_{A}$ the set of "self-loops" in $\mathcal{H}$, namely $\mathcal{E}_{\text {loop }}=\left\{\left(x_{i}, x_{i}^{\prime}\right)\right\}_{i=1}^{N} \cap$ $\overline{\mathcal{E}}_{A}$. Then, we define $\mathcal{E}_{\text {new }}$ as the set of "self-loops" that are not present in $\mathcal{H}$, namely $\mathcal{E}_{\text {new }}=\left\{\left(x_{i}, x_{i}^{\prime}\right)\right\}_{i=1}^{N} \backslash \overline{\mathcal{E}}_{A}$. With this notation, $\mathcal{H}_{\times}$is obtained from $\mathcal{H}$ by adding all new "self-loops", i.e., the edge set of $\mathcal{H}_{\times}$is $\overline{\mathcal{E}}_{A} \cup \overline{\mathcal{E}}_{B} \cup \overline{\mathcal{E}}_{C} \cup$ $\overline{\mathcal{E}}_{D} \cup \mathcal{E}_{\text {new }}$. Notice that edges in $\overline{\mathcal{E}}_{A} \cup \mathcal{E}_{\text {loop }}$ are in one-toone correspondence with entries of the matrix pencil $A-z I$ which are non-zero, i.e., which are either $a_{i j}$ with $j \neq i$ (edge in $\overline{\mathcal{E}}_{A}$ which is not a "self-loop"), or $a_{i i}-z$ (edge in $\mathcal{E}_{\text {loop }}$ ), or $-z$ (edge in $\mathcal{E}_{\text {new }}$ ).

The last construction that we need is the bipartite graph $\mathcal{N}$. Its left vertex set is $U_{0} \cup X \cup U_{1}$ and its right vertex set is $Y_{0} \cup X^{\prime} \cup Y_{1}$, where $X$ and $X^{\prime}$ are the same as above, $U_{0}=\left\{u_{1}^{0}, \ldots, u_{P}^{0}\right\}$ and $U_{1}=\left\{u_{1}^{1}, \ldots, u_{P}^{1}\right\}$ are two copies of the input vertex set, and $Y_{0}=\left\{y_{1}^{0}, \ldots, y_{M}^{0}\right\}$ and $Y_{1}=$ $\left\{y_{1}^{1}, \ldots, y_{M}^{1}\right\}$ are two copies of the output vertex set. The edge set of $\mathcal{N}$ is $\mathcal{F}_{D}^{0} \cup \mathcal{F}_{D}^{1} \cup \mathcal{F}_{B} \cup \mathcal{F}_{C} \cup \mathcal{F}_{I}$, where:

- $\mathcal{F}_{D}^{h}=\left\{\left(u_{j}^{h}, y_{i}^{h}\right) \in U_{h} \times Y_{h}\right.$ s.t. $\left.D_{i j} \neq 0\right\}$, for $h=0,1$,

- $\mathcal{F}_{B}=\left\{\left(u_{j}^{0}, x_{i}^{\prime}\right) \in U_{0} \times X^{\prime}\right.$ s.t. $\left.B_{i j} \neq 0\right\}$,

- $\mathcal{F}_{C}=\left\{\left(x_{j}, y_{i}^{1}\right) \in X \times Y_{1}\right.$ s.t. $\left.C_{i j} \neq 0\right\}$, and

- $\mathcal{F}_{I}=\left\{\left(x_{j}, x_{i}^{\prime}\right) \in X \times X^{\prime}\right.$ s.t. $\left.j=i\right\}$.

Finally, we will also use two particular subgraphs of $\mathcal{N}$ : first, $\tilde{\mathcal{N}}$, obtained from $\mathcal{N}$ by removing the vertices in $U_{1}$ and the edges in $\mathcal{F}_{D}^{1}$; then, $\mathcal{N}_{0}$, obtained from $\tilde{\mathcal{N}}$ by further removing the vertices in $Y_{0}$ and the edges in $\mathcal{F}_{D}^{0}$.

Figures $1 \mathrm{a}$ and 2 are sketches that pictorially remind the above-described constructions, while Figures $1 \mathrm{~b}$ and 3 illustrate the following Example 1. 


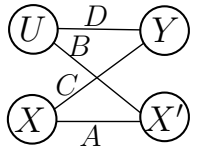

(a) $\mathcal{H}$

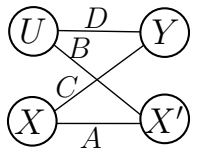

(b) $\mathcal{H}_{\times}$

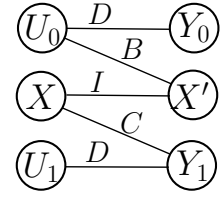

(c) $\mathcal{N}$
Fig. 2: Sketch of the construction of $\mathcal{H}, \mathcal{H}_{\times}$, and $\mathcal{N}$. From $\mathcal{N}, \tilde{\mathcal{N}}$ is obtained by removing $U_{1}$ and its incident edges, and $\mathcal{N}_{0}$ by removing $U_{1}, Y_{0}$ and their incident edges.

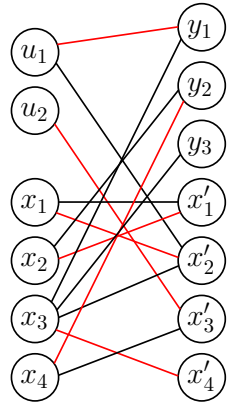

(a) $\mathcal{H}$

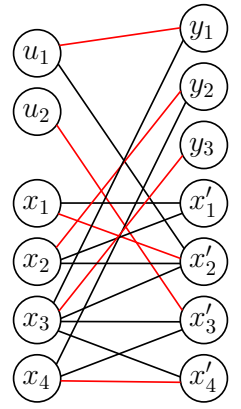

(b) $\mathcal{H}_{\times}$

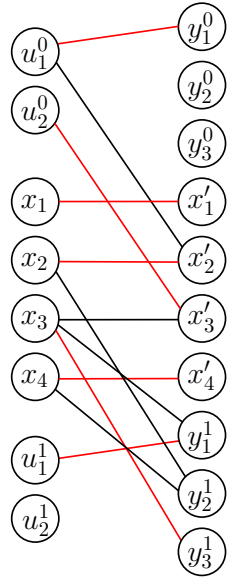

(c) $\mathcal{N}$
Fig. 3: Bipartite graphs $\mathcal{H}, \mathcal{H}_{\times}, \mathcal{N}$ for Example 1. In $\mathcal{H}_{\times}$, $\mathcal{E}_{\text {loop }}=\left\{\left(x_{1}, x_{1}^{\prime}\right)\right\}$ and $\mathcal{E}_{\text {new }}=\left\{\left(x_{2}, x_{2}^{\prime}\right),\left(x_{3}, x_{3}^{\prime}\right),\left(x_{4}, x_{4}^{\prime}\right)\right\}$. Red edges represent the uniquely restricted matchings $\mathcal{M}$, $\mathcal{M} \times$ and $\mathcal{L}$ described in Sect. IV.

Example 1: Consider the structured system (1) with the following matrices:

$$
\begin{array}{ccc}
A & =\left[\begin{array}{cccc}
a_{11} & a_{12} & 0 & 0 \\
a_{21} & 0 & a_{23} & 0 \\
0 & 0 & 0 & a_{34} \\
0 & 0 & a_{43} & 0
\end{array}\right], & B=\left[\begin{array}{cc}
0 & 0 \\
b_{21} & 0 \\
0 & b_{32} \\
0 & 0
\end{array}\right], \\
C=\left[\begin{array}{cccc}
0 & 0 & c_{13} & \\
0 & c_{22} & 0 & c_{24} \\
0 & 0 & c_{33} & 0
\end{array}\right], \quad D=\left[\begin{array}{cc}
d_{11} & 0 \\
0 & 0 \\
0 & 0
\end{array}\right] .
\end{array}
$$

For this example, Figure $1 \mathrm{~b}$ shows the directed graph $\mathcal{G}$, while Figure 3 shows the bipartite graphs $\mathcal{H}, \mathcal{H}_{\times}$, and $\mathcal{N}$. Since the only self-loop in $\mathcal{G}$ is on $x_{1}$, we have $\mathcal{E}_{\text {loop }}=$ $\left\{\left(x_{1}, x_{1}^{\prime}\right)\right\}$; the other "self-loops" are new in $\mathcal{H}_{\times}$and form the set $\mathcal{E}_{\text {new }}=\left\{\left(x_{2}, x_{2}^{\prime}\right),\left(x_{3}, x_{3}^{\prime}\right),\left(x_{4}, x_{4}^{\prime}\right)\right\}$. The bipartite graphs $\tilde{\mathcal{N}}$ and $\mathcal{N}_{0}$ are not illustrated in the figure. The former is the subgraph of $\mathcal{N}$ obtained by removing the vertices $u_{1}^{1}$, $u_{2}^{1}$ and the edge $\left(u_{1}^{1}, y_{1}^{1}\right)$. Then, $\mathcal{N}_{0}$ is obtained by further removing the vertices $y_{1}^{0}, y_{2}^{0}, y_{3}^{0}$ and the edge $\left(u_{1}^{0}, y_{1}^{0}\right)$.

\section{B. Structured Matrix and Associated Bipartite Graph}

In this paper, we will use the following definitions (note that different vocabulary is used by different authors). We say that a matrix $S$ is a structured matrix if its entries are real polynomials in some variables, say $\lambda_{1}, \ldots, \lambda_{\mu}$. We consider the variables as real-valued parameters. We say that a structured matrix $S$ is a pattern matrix if all its non-zero entries are of the form $\lambda_{i}$, with all $i$ 's being distinct. With this vocabulary, matrices $A, B, C$, and $D$ of the structured system are pattern matrices, while some structured matrices not being pattern matrices will appear in the characterizations of (unconstrained) ISO.

It is customary [8] to define the bipartite graph $\mathcal{B}(S)$ associated to the structured matrix $S$ in the following manner: the left vertex set is the set of all columns of $S$, the right vertex set is the set of all rows of $S$, and there is an edge from column $j$ to row $i$ if and only if $S_{i j}$ is a non-zero polynomial.

Recall that a matching is a collection of edges such that no two edges share a common vertex; its size is the number of edges. The term rank of a structured matrix $S$, denoted term-rank $(S)$, is defined as the size of the maximum matching in $\mathcal{B}(S)$. For any choice of the parameters, $\operatorname{rank} S \leq$ term-rank $S$ (see e.g. [8, Chapter 2]).

The notion of uniquely restricted matching (also known as constrained matching), which underpins many strongly structural results, is defined as follows.

Definition $4([9$, Definition 2.4]): Let $\mathcal{B}$ be a bipartite graph, with left vertex set $V$, right vertex set $W$, and edge set $\mathcal{E}$. A matching $\mathcal{M} \subseteq \mathcal{E}$ of size $s$ is uniquely restricted if there exists no other matching $\mathcal{M}^{\prime} \subseteq \mathcal{E}$ covering exactly the same set of vertices.

The following lemma gives a useful characterization of uniquely restricted matchings.

Lemma 1 ([10, Thm. 3.1]): Let $\mathcal{B}$ be a bipartite graph, with left vertex set $V$, right vertex set $W$, and edge set $\mathcal{E}$. A matching $\mathcal{M} \subseteq \mathcal{E}$ of size $s$ is uniquely restricted if and only if there exists a reordering of vertices $V=\left\{v_{1}, \ldots, v_{|V|}\right\}$ and $W=\left\{w_{1}, \ldots, w_{|W|}\right\}$ such that $\mathcal{M}=\left\{\left(v_{i}, w_{i}\right)\right\}_{i=1}^{s}$ and moreover $\left(v_{j}, w_{i}\right) \notin \mathcal{E}$ for $1 \leq i<j \leq s$.

The following result and the techniques introduced in [9] for its proof are of major importance for strongly structural results.

Lemma 2 ([9, Thm. 3.9]): If $S$ is a structured matrix, then the following are equivalent:

- $\operatorname{rank} S=r$ for all non-zero parameters;

- term-rank $S=r$ and there exists a uniquely restricted matching of size $r$ in $\mathcal{B}(S)$.

\section{MAIN RESUlT}

In this section we present the statement of our main results, presenting graphical conditions for s-structural (unconstrained) ISO, together with a discussion of the algorithms that can be used to check such conditions and of their complexity. Proofs are postponed to next sections.

The following theorem gives a graphical characterization of s-structural unconstrained ISO. This is the counterpart for unconstrained ISO of the characterization of controllability given in [3, Theorem 5].

Theorem 1: The structured system (1) is s-structurally unconstrained ISO if and only if the following conditions are satisfied: 
(i) there exists a uniquely restricted matching $\mathcal{M}$ of size $P+N$ in the bipartite graph $\mathcal{H}$, and

(ii) there exists a uniquely restricted matching $\mathcal{M}_{\times}$of size $P+N$ in the bipartite graph $\mathcal{H}_{\times}$such that $\mathcal{M}_{\times} \cap$ $\mathcal{E}_{\text {loop }}=\emptyset$.

See Section V for the proof of Theorem 1.

The following result gives a sufficient condition and a necessary condition for s-structural ISO. ISO:

Theorem 2: The structured system (1) is s-structurally

- if it is s-structurally unconstrained ISO and there exists a uniquely restricted matching of size $P+N+$ term-rank $(D)$ in the bipartite graph $\mathcal{N}$;

- only if it is s-structurally unconstrained ISO and there exists a uniquely restricted matching of size $P+N$ in the bipartite graph $\tilde{\mathcal{N}}$.

See Section VI for the proof of Theorem 2.

In general, there is a gap between the necessary condition and the sufficient condition in Theorem 2. However, in the case where there is no direct feedthrough of the input to the output $(D=0)$ the two conditions are equivalent and give the following characterization of s-structural ISO.

Corollary 1: If $D=0$, the structured system (1) is sstructurally ISO if and only if it is s-structurally unconstrained ISO and there exists a uniquely restricted matching of size $P+N$ in the bipartite graph $\mathcal{N}_{0}$.

Proof: Since $D=0, \mathcal{F}_{D}^{1}=\mathcal{F}_{D}^{2}=\emptyset$ and hence the three bipartite graphs $\mathcal{N}, \tilde{\mathcal{N}}$, and $\mathcal{N}_{0}$ have the same edges and only differ by some stranded vertices, which are irrelevant for the existence of a uniquely restricted matching of some required size. Finally notice that $D=0$ implies term-rank $D=0$, so that the required size is $P+N$ both in the necessity and in the sufficiency part. $\square$

The following remark describes the algorithms that can be used to check the conditions in our characterizations, and discusses their complexity.

Remark 1: Note that all the conditions given in Theorem 1, Theorem 2 and Corollary 1 are questions of the following kind: In a given bipartite graph, for some given set of edges, is there a maximum matching of a given size, only using the given edges, and which is also uniquely restricted? Corollary 4 in [11] provides an algorithm to answer this question in polynomial time. In all above-mentioned conditions, except the sufficiency part of Theorem 2, the given size to be considered is the size of the left vertex set. For this specific size, a simpler algorithm has been presented in [12] and refined in [3], which is equivalent to the one in [11] particularized to this case. A clever implementation of the same algorithm, devised by [13], has a complexity which is linear in the number of vertices plus the number of edges; depending on the sparsity level of the system matrices, this is in between linear and quadratic w.r.t. $N+P+M$.

Example 1 (Continued): We will now show that the structured system in Example 1 is s-structurally ISO. First, we construct the following matching $\mathcal{M}$ in $\mathcal{H}$ (see Fig. 3a): $\mathcal{M}=\left\{\left(u_{1}, y_{1}\right),\left(u_{2}, x_{3}^{\prime}\right),\left(x_{1}, x_{2}^{\prime}\right),\left(x_{2}, x_{1}^{\prime}\right),\left(x_{3}, x_{4}^{\prime}\right)\right.$, $\left.\left(x_{4}, y_{2}\right)\right\}$. Notice that $\mathcal{M}$ has size $6=P+N$, and it is uniquely constrained in $\mathcal{H}$, since there is no other matching in $\mathcal{H}$ covering the same set of vertices. Since $\mathcal{H}$ is a subgraph of $\mathcal{H}_{\times}, \mathcal{M}$ is also a matching in $\mathcal{H}_{\times}$. However, $\mathcal{M}$ is not uniquely constrained in $\mathcal{H}_{\times}$. Indeed, by replacing edges $\left(x_{1}, x_{2}^{\prime}\right)$ and $\left(x_{2}, x_{1}^{\prime}\right)$ with edges $\left(x_{1}, x_{1}^{\prime}\right)$ and $\left(x_{2}, x_{2}^{\prime}\right)$ we obtain another matching in $\mathcal{H}_{\times}$covering the same vertices as $\mathcal{M}$. The following matching $\mathcal{M}_{\times}$, instead, is uniquely restricted in $\mathcal{H}_{\times}$(see Fig. 3b): $\mathcal{M}_{\times}=\left\{\left(u_{1}, y_{1}\right),\left(u_{2}, x_{3}^{\prime}\right)\right.$, $\left.\left(x_{1}, x_{2}^{\prime}\right),\left(x_{2}, y_{2}\right),\left(x_{3}, y_{3}\right),\left(x_{4}, x_{4}^{\prime}\right)\right\}$. Notice that $\mathcal{M}_{\times}$has size $P+N$ and $\mathcal{M}_{\times} \cap E_{\text {loop }}=\emptyset$. By Theorem 1, we have obtained that this system is s-structurally unconstrained ISO. To ensure that it is also s-structurally ISO, we study $\mathcal{N}$. The following matching $\mathcal{L}$ is uniquely restricted in $\mathcal{N}$ (see Fig. 3c): $\mathcal{L}=\left\{\left(u_{1}^{0}, y_{1}^{0}\right),\left(u_{2}^{0}, x_{3}^{\prime}\right),\left(x_{1}, x_{1}^{\prime}\right),\left(x_{2}, x_{2}^{\prime}\right)\right.$, $\left.\left(x_{3}, y_{3}^{1}\right),\left(x_{4}, x_{4}^{\prime}\right),\left(u_{1}^{1}, y_{1}^{1}\right)\right\}$. Notice that term-rank $(D)=1$ and that $\mathcal{L}$ has size $7=P+N+$ term-rank $(D)$. Hence, Theorem 2 ensures that this system is s-structurally ISO.

\section{Proof of Theorem 1}

The proof of Theorem 1 in inspired from the proofs of the characterizations of s-structural controllability in [2] and [3], and uses suitable modifications of the proofs in [9]. Our proof is based on the following PBH-like algebraic characterization of unconstrained ISO, which involves the matrix pencil

$$
P(z)=\left[\begin{array}{cc}
A-z I_{N} & B \\
C & D
\end{array}\right] .
$$

Lemma 3 ([14, Theorems 2.7 and 2.8]): System (1) is unconstrained ISO if and only if the corresponding matrix pencil $P(z)$ satisfies $\operatorname{rank}(P(z))=N+P$ for all $z \in \mathbb{C}$.

\section{A. Proof of Sufficiency}

A key tool in the development of s-structural results involves transforming matrices into suitable triangular forms. We adopt this classical technique and we introduce the following Form IV, following the definition of Forms I, II and III in the literature (see e.g. [2]).

Definition 5: A matrix $S$ is said to be in Form IV if

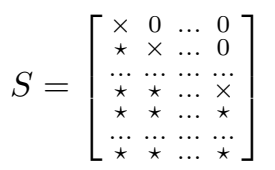

where $x$ denote entries which are not a zero, 0 denote positions fixed to zero and $\star$ denote positions that could be either zero or non-zero.

The following lemma gives a sufficient condition for sstructural unconstrained ISO in terms of transformation of matrix pencil $P(z)$ into Form IV.

Lemma 4: The structured system (1) is s-structurally unconstrained ISO if the following two conditions hold:

(i) there exist permutation matrices $P_{1}, P_{2}$ such that the matrix

is in Form IV;

$$
P_{1}\left[\begin{array}{ll}
A & B \\
C & D
\end{array}\right] P_{2}
$$

(ii) there exist permutation matrices $P_{3}, P_{4}$ such that the matrix 


$$
P_{3}\left[\begin{array}{cc}
A-z I_{N} & B \\
C & D
\end{array}\right] P_{4}
$$

is in Form IV, and moreover none of the $\times$-terms is $a_{i i}-z$ for some $i$, i.e., each $\times$-term is either -1 or a free parameter.

Proof: Condition (i) ensures that $\operatorname{rank} P(0)=N+P$ for all non-zero parameters. Indeed, since $P(0)=\left[\begin{array}{ll}A & B \\ C & D\end{array}\right]$, by condition (i) we know that $P_{1} P(0) P_{2}$ is in Form IV. We look at the square submatrix of size $N+P$ of $P_{1} P(0) P_{2}$ which is triangular, with diagonal terms $\times$. Such diagonal terms are all free parameters, and hence any choice of non-zero parameters gives non-zero determinant of this submatrix, so that $\operatorname{rank} P(0)=\operatorname{rank}\left(P_{1} P(0) P_{2}\right)=N+P$.

Condition (ii) ensures that $\operatorname{rank} P(z)=N+P$ for all non-zero parameters and all $z \neq 0$. We look at the square submatrix of size $N+P$ of $P_{3} P(z) P_{4}$ having diagonal terms $\times$; such diagonal terms are ensured to be either free parameters or $-z$, not $a_{i i}-z$. Thus any choice of non-zero parameters and non-zero $z$ gives non-zero determinant of this submatrix and $\operatorname{rank} P(z)=\operatorname{rank}\left(P_{3} P(z) P_{4}\right)=N+P$.

The algebraic conditions given in Lemma 4 can be transformed in the graph-theoretic terms used in Theorem 1, thanks to the following remark.

Remark 2: Let $S$ be a structured matrix with $s$ columns, and let $\mathcal{B}(S)$ be the associated bipartite graph. Comparing the definition of Form IV and the characterization of uniquely restricted matchings given in Lemma 1, we can see that there exists a uniquely restricted matching of size $s$ in $\mathcal{B}(S)$ if and only if there exist permutation matrices $P_{1}, P_{2}$ such that $P_{1} S P_{2}$ is in Form IV; the $\times$-terms of the Form IV correspond to the edges of the uniquely restricted matching. Indeed, right-multiplication by $P_{2}$ permutes the columns of $S$, which is equivalent to a re-ordering of the left vertex set of $\mathcal{B}(S)$, and left-multiplication by $P_{1}$ permutes the rows of $S$, which is equivalent to a re-ordering of the right vertex set of $\mathcal{B}(S)$. Under this re-ordering, the statement that $P_{1} S P_{2}$ is in Form IV is equivalent to the fact that there is a matching $\mathcal{M}=\left\{\left(v_{1}, w_{1}\right), \ldots,\left(v_{s}, w_{s}\right)\right\}$, since these edges correspond to the $\times$-terms of $P_{1} S P_{2}$ and ensure that they are non-zero, together with the condition $\left(v_{j}, w_{i}\right) \notin \mathcal{E}_{S}$ if $1 \leq j<i \leq s$, which means that all entries above the $\times$-terms are zeros.

Notice that $\mathcal{H}$ is the bipartite graph associated with $P(0)=\left[\begin{array}{ll}A & B \\ C & D\end{array}\right]$ and $\mathcal{H}_{\times}$is the bipartite graph associated with $P(z)$. By applying Remark 2 to $\mathcal{H}$ and $\mathcal{H}_{\times}$, we obtain that conditions (i) and (ii) in the statement of Theorem 1 imply conditions (i) and (ii), respectively, in Lemma 4. The latter comes from the fact that the edges in the matching $\mathcal{M}_{\times}$not being from $\mathcal{E}_{\text {loop }}$ ensures that the $\times$-terms in the Form IV are not polynomials $a_{i i}-z$ for some free parameter $a_{i i}$.

\section{B. Proof of Necessity}

We show necessity of conditions (i) and (ii) in Theorem 1. Condition (i) is necessary to ensure that $P(0)$ has full column rank for all non-zero parameters. Indeed, $P(0)=$ $\left[\begin{array}{ll}A & B \\ C & D\end{array}\right]$ is a pattern matrix, with bipartite graph $\mathcal{B}(P(0))=\mathcal{H}$. By Lemma 2, if there is no uniquely restricted matching of size $N+P$ in $\mathcal{H}$, then $P(0)$ cannot have full column rank
$N+P$ for all non-zero parameters; by Lemma 3 this also means that this choice of parameters gives a system which is not unconstrained ISO.

Condition (ii), instead, concerns the rank of $P(z)$ when $z \neq 0$. In particular, in the remainder of this section we will focus on $P(1)=\left[\begin{array}{cc}A-I & B \\ C & D\end{array}\right]$, and we will show that condition (ii) is necessary to ensure that $P(1)$ has full column rank for all non-zero parameters. More precisely, we will prove the following lemma, which gives the desired statement by setting $r=N+P$, but has a formulation with general $r$, more suitable for the proof by induction. In this lemma, we will consider the submatrices of $P(1)$ formed by selecting $r$ columns of $P(1)$, and the associated bipartite graphs, which are the subgraphs of $\mathcal{H}_{\times}$having left vertex set reduced to the $r$ vertices corresponding to the selected columns.

Lemma 5: Let $r$ be an integer, $1 \leq r \leq N+P$. For any matrix $P_{r}$ formed with $r$ columns of $P(1)$, if there does not exist a uniquely restricted matching $\mathcal{M}_{\times}^{(r)}$ of size $r$ in $\mathcal{B}\left(P_{r}\right)$ that satisfies $\mathcal{M}_{\times}^{(r)} \cap \mathcal{E}_{\text {loop }}=\emptyset$, then wither $P_{r}=0$ or there exists non-zero parameters such that the corresponding numerical realization of $P_{r}$ satisfies rank $P_{r}<r$.

Proof: The base case is $r=1$, where $P_{1}$ is a column of $P(1)$ (say the $i$-th column) and hence $\mathcal{B}\left(P_{1}\right)$ has only one vertex in its left vertex set. This implies that each edge is a matching of size 1 , and is also a uniquely restricted matching. Hence, if there does not exist a uniquely restricted matching in $\mathcal{B}\left(P_{1}\right)$ with empty intersection with $\mathcal{E}_{\text {loop }}$, then all edges of $\mathcal{B}\left(P_{1}\right)$ are in $\mathcal{E}_{\text {loop. }}$ This means that $P_{1}$ is either the allzero vector, or a vector with a unique non-zero entry, which is equal to $a_{i i}-1$ since the corresponding edge is in $\mathcal{E}_{\text {loop }}$. In the latter case, we can set $a_{i i}=1$ and notice that this non-zero parameter corresponds to $P_{1}=0$, which implies $\operatorname{rank} P_{1}=0<r=1$.

Now we assume that the claim holds for $r-1$ (inductive assumption) and we prove that this implies that the claim holds for $r$. There are two cases, that require different proofs.

Case a): there exists a row of $P_{r}$ having exactly one non-zero entry, and such that this non-zero entry is not of the form $a_{i i}-1$, i.e., it is either a free parameter, or -1 . Say that this is the $k$-th row, and its non-zero entry is in position $(k, \ell)$. Denote by $P_{r-1}$ the submatrix of $P_{r}$ obtained by removing the $\ell$-th column. Notice that $P_{r-1}$ is a matrix formed with $r-1$ columns of $P(1)$. If there is no uniquely restricted matching of size $r$ in $\mathcal{B}\left(P_{r}\right)$ with empty intersection with $\mathcal{E}_{\text {loop }}$, then there is no uniquely restricted matching of size $r-1$ in $\mathcal{B}\left(P_{r-1}\right)$ with empty intersection with $\mathcal{E}_{\text {loop }}$; indeed, if the latter existed, then one would obtain the former simply by adding the edge corresponding to the $(k, \ell)$-th entry of $P_{r}$. Hence, by inductive assumption applied to $P_{r-1}$, either $P_{r-1}$ or there exists some non-zero parameters such that the corresponding numerical realization of $P_{r-1}$ has rank $P_{r-1}<r-1$. With the same parameters (if any), together with an arbitrary non-zero value for the $(k, \ell)$ th entry in case it is a free parameter, we have a choice of non-zero parameters such that rank $P_{r}<r$. 
Case $b$ ): the complement of case a). This means that all rows of $P_{r}$ fall in the following categories: 1) all-zero row; 2) a row with $p \geq 1$ non-zero entries, one of which of the form $a_{i i}-1$ for some $i$, and $p-1$ being a free parameter; 3 ) a row with $p \geq 2$ non-zero entries, being either $p$ free parameters, or one -1 and $p-1$ free parameters. If $P_{r} \neq 0$, we can find non-zero parameters such that the corresponding numerical evaluation $P_{r}$ has all row-sums equal to zero. Rows in the first category already have zero sum. For rows in the second category, we can choose $a_{i i}=p$ and all other parameters (if any) equal to -1 . For rows in the third category, we can choose one free parameter equal to $p-1$ and all other free parameters (if any) equal to -1 . With this choice, the sum of the columns of $P_{r}$ is the zero vector, which implies that rank $P_{r}<r$.

\section{PROOF OF THEOREM 2}

Recall the following classical definition of delay-1 left invertibility (see e.g. [14, Definition 2.5]).

Definition 6: System (1) is delay-1 left invertible if the unknown input $u_{0}$ is uniquely determined by the initial state $x_{0}$ and the outputs $y_{0}, y_{1}$.

Notice that ISO is equivalent to unconstrained ISO together with delay-1 left invertibility. Hence, to prove Theorem 2, we only need prove that the two graphical conditions mentioned in Theorem 2 are respectively sufficient and necessary for s-structural delay-1 left invertibility. Our proof relies on the following algebraic characterization of delay-1 left invertibility.

Lemma 6 ([15, Prop. 2]): Define

$$
Q=\left[\begin{array}{ccc}
D & 0 & 0 \\
B & -I & 0 \\
0 & C & D
\end{array}\right]
$$

System (1) is delay-1 left invertible if and only if $\operatorname{rank} Q=P+N+\operatorname{rank} D$.

To prove the sufficiency part of Theorem 2 , let $R$ be a matrix obtained from $Q$ by replacing each -1 with a free parameter, and replacing each parameter appearing twice (due to the two submatrices equal to $D$ ) with two distinct parameters. Notice that $R$ is a pattern matrix, and $\mathcal{N}=\mathcal{B}(R)$. Since clearly term-rank $(R) \leq P+N+\operatorname{term}-\operatorname{rank}(D)$, by Lemma 2 , if there exists a uniquely restricted matching of size $P+N+\operatorname{term}-\operatorname{rank}(D)$ in $\mathcal{N}$, then $\operatorname{rank} R=$ $P+N+\operatorname{term}-\operatorname{rank}(D)$ for all non-zero parameters, and hence in particular $\operatorname{rank}(Q)=P+N+\operatorname{term-rank}(D)$ for all non-zero parameters. Moreover, since for any choice of parameters rank $D \leq \operatorname{term-rank} D$, this further implies that $\operatorname{rank}(Q)=P+N+\operatorname{rank}(D)$ for all non-zero parameters, so that by Lemma 6 the system is s-structurally delay-1 left invertible.

To prove the necessity part of Theorem 2 , let $\tilde{Q}$ be the submatrix of $Q$ formed with the first $P+N$ columns and notice that $\tilde{\mathcal{N}}=\mathcal{B}(\tilde{Q})$. First, notice that Lemma 6 implies that $\operatorname{rank}(\tilde{Q})=P+N$ is a necessary condition for delay-1 left invertibility. Then, we can prove the following lemma, which gives the desired result when taking $r=P+N$.
Lemma 7: Let $r$ be an integer, $1 \leq r \leq P+N$. For any submatrix $\tilde{Q}_{r}$ formed with $r$ columns of $\tilde{Q}$, if there does not exist a uniquely restricted matching of size $r$ in $\mathcal{B}\left(\tilde{Q}_{r}\right)$, then either $\tilde{Q}_{r}=0$ or there exist non-zero parameters such that the corresponding realization of $\tilde{Q}_{r}$ has $\operatorname{rank} \tilde{Q}_{r}<r$.

The proof by induction is the same as the proof of Lemma 5 in the simple case where $\mathcal{E}_{\text {loop }}=\emptyset$ and hence is omitted.

\section{CONCLUSION}

In the present paper, we have studied s-structural (unconstrained) ISO for LTI network systems. First, we have provided a graphical characterization for s-structural unconstrained ISO. Then we have found a sufficient condition and a necessary condition for s-structural ISO. Under the assumption of no direct feedthrough, the said conditions can be combined to fully characterize s-structural ISO, while the characterization in the presence of direct feedthrough remains as an open problem. All our graphical conditions are in terms of existence of suitable uniquely restricted matchings in some bipartite graphs, and can be verified in polynomial time with respect to the dimension of the input, state and output spaces.

\section{REFERENCES}

[1] H. Mayeda and T. Yamada, "Strong structural controllability," SIAM J. Control Optim., vol. 17, no. 1, pp. 123-138, 1979.

[2] J. C. Jarczyk, F. Svaricek, and B. Alt, "Strong structural controllability of linear systems revisited," in Proc. 50th IEEE Conf. Decision Control and European Control Conf. (CDC-ECC), Orlando, Florida, 2011, pp. 1213-1218.

[3] A. Chapman and M. Mesbahi, "On strong structural controllability of networked systems: a constrained matching approach," in Proc. 2013 American Control Conf., Washington, DC, USA, 2013, pp. 6126-6131.

[4] N. Monshizadeh, S. Zhang, and M. K. Camlibel, "Zero forcing sets and controllability of dynamical systems defined on graphs," IEEE Trans. Autom. Control, vol. 59, no. 9, pp. 2562-2567, 2014.

[5] M. Trefois and J.-C. Delvenne, "Zero forcing number, constrained matchings and strong structural controllability," Linear Algebra Appl., vol. 484, pp. 199-218, 2015.

[6] S. Gracy, F. Garin, and A. Y. Kibangou, "Structural and strongly structural input and state observability of linear network systems," IEEE Trans. Control Netw. Syst., vol. 5, no. 4, pp. 2062-2072, 2018

[7] — " "Input and state observability of network systems with timevarying topology," IEEE Trans. Control Netw. Syst., 2019, in press.

[8] K. Murota, Matrices and matroids for systems analysis. SpringerVerlag, 2000.

[9] D. Hershkowitz and H. Schneider, "Ranks of zero patterns and sign patterns," Linear Multilinear Algebra, vol. 34, no. 1, pp. 3-19, 1993.

[10] M. C. Golumbic, T. Hirst, and M. Lewenstein, "Uniquely restricted matchings," Algorithmica, vol. 31, no. 2, pp. 139-154, 2001.

[11] L. D. Penso, D. Rautenbach, and U. dos Santos Souza, "Graphs in which some and every maximum matching is uniquely restricted," Journal of Graph Theory, 2015.

[12] C. Hartung, G. Reißig, and F. Svaricek, "Characterization of strong structural controllability of uncertain linear time-varying discrete-time systems," in 51st IEEE Conf. Decision Control (CDC), Maui, Hawaii, USA, 2012, pp. 2189-2194.

[13] A. Weber, G. Reißig, and F. Svaricek, "A linear time algorithm to verify strong structural controllability," in Proc. 53rd IEEE Conf. Decision Control (CDC), Los Angeles, CA, USA, 2014, pp. 55745580.

[14] S. Sundaram, "Fault-tolerant and secure control systems," University of Waterloo, Lecture Notes, 2012. [Online]. Available: https: //engineering.purdue.edu/ $\sim$ sundara $2 / \mathrm{misc} / \mathrm{ft}$ _control_lecture_notes.pdf

[15] F. Garin, "Structural delay-1 input-and-state observability," in Proc. 56th IEEE Conf. Decision Control (CDC), Melbourne, Australia, 2017, pp. $2324-2329$. 